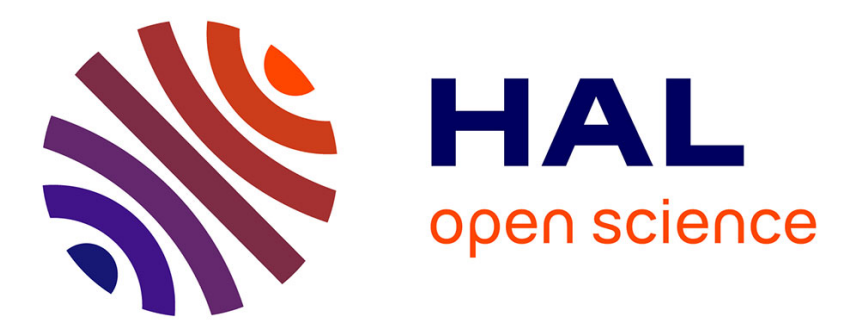

\title{
A Natural Formalism and a MultiAgent Algorithm for Integrative Multidisciplinary Design Optimization
}

\author{
Tom Jorquera, Jean-Pierre Georgé, Marie-Pierre Gleizes, Christine Régis
}

\section{To cite this version:}

Tom Jorquera, Jean-Pierre Georgé, Marie-Pierre Gleizes, Christine Régis. A Natural Formalism and a MultiAgent Algorithm for Integrative Multidisciplinary Design Optimization. IEEE/WIC/ACM International Conference on Intelligent Agent Technology (IAT 2013), Nov 2013, Atlanta, United States. pp.146-154, 10.1109/WI-IAT.2013.103 . hal-01148099

\section{HAL Id: hal-01148099 \\ https://hal.science/hal-01148099}

Submitted on 4 May 2015

HAL is a multi-disciplinary open access archive for the deposit and dissemination of scientific research documents, whether they are published or not. The documents may come from teaching and research institutions in France or abroad, or from public or private research centers.
L'archive ouverte pluridisciplinaire HAL, est destinée au dépôt et à la diffusion de documents scientifiques de niveau recherche, publiés ou non, émanant des établissements d'enseignement et de recherche français ou étrangers, des laboratoires publics ou privés. 


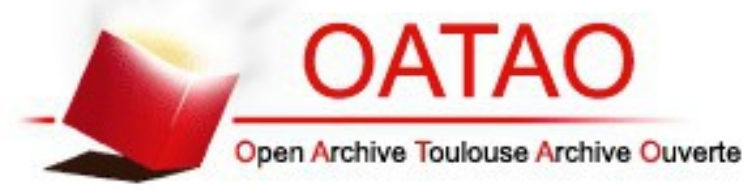

\section{Open Archive TOULOUSE Archive Ouverte (OATAO)}

OATAO is an open access repository that collects the work of Toulouse researchers and makes it freely available over the web where possible.

This is an author-deposited version published in : http://oatao.univ-toulouse.fr/ Eprints ID : 12859

To link to this article : DOI :10.1109/WI-IAT.2013.103

URL : http://dx.doi.org/10.1109/WI-IAT.2013.103

To cite this version : Jorquera, Tom and Georgé, Jean-Pierre and Gleizes, Marie-Pierre and Régis, Christine $\underline{A \text { Natural Formalism and } a}$ MultiAgent Algorithm for Integrative Multidisciplinary Design Optimization. (2013) In: IEEE/WIC/ACM International Conference on Intelligent Agent Technology - IAT 2013, 17 November 2013 - 20 November 2013 (Atlanta, United States).

Any correspondance concerning this service should be sent to the repository administrator: staff-oatao@,listes-diff.inp-toulouse.fr 


\title{
A Natural Formalism and a Multi-Agent Algorithm for Integrative Multidisciplinary Design Optimization
}

\author{
Tom Jorquera, Jean-Pierre Georgé, Marie-Pierre Gleizes, Christine Régis \\ IRIT \\ University of Toulouse \\ France \\ Email: \{jorquera,george,gleizes,regis $\} @$ irit.fr
}

\begin{abstract}
MultiDisciplinary Optimization (MDO) problems represent one of the hardest and broadest domains of continuous optimization. By involving both the models and criteria of different disciplines, MDO problems are often too complex to be tackled by classical optimization methods. We propose an approach which takes into account this complexity using a new representation (NDMO - Natural Domain Modeling for Optimization) and a self-adaptive multi-agent algorithm. Our method agentifies the different elements of the problem (such as the variables, the models, the objectives). Each agent is in charge of a small part of the problem and cooperates with others to find equilibrium on conflicting values. Despite the fact that no agent of the system has a complete view of the entire problem, the mechanisms we provide allow the emergence of a coherent solution. Evaluations on several academic and industrial test cases are provided.
\end{abstract}

Keywords-Multi-Agent System, Multidisciplinary Optimization, Integrative Design

\section{INTRODUCTION}

In their review about MultiDisciplinary Optimization (MDO), Sobieszczansky-Sobieski and Haftka propose to define it as methodology for the design of systems in which strong interaction between disciplines motivates designers to simultaneously manipulate variables in several disciplines [1]. Designers have to simultaneously consider different disciplines (such as, for example, aerodynamics, geometrics and acoustics for an aircraft engine) which are often not only complex by themselves but also strongly interdependent, causing the classical optimization approaches to struggle handling them.

Formally, MDO problems are continuous optimization problems where the goal is to find the values of a set of inputs that maximize (or minimize) several objectives while satisfying several constraints (both often regrouped under the term optimization criteria). These problems tend to be complex to solve as they can involve calculus-heavy, interdependent models and contradictory criteria.

Currently, MDO problems require specific strategies to be solved, and a major part of the research in the field has been focusing on providing these strategies. These approaches often involve reformulating the problem, requiring techniques to maintain coherency among variables shared by different disciplines and specific ordering of local optimizations on subparts of the problem. Thus an important part of the burden is still on the shoulders of the engineers.
In this paper, we propose an original approach using a Multi-Agent System (MAS) [2] for solving this kind of optimization problem in the most generic way while keeping the need to reformulate the problem at a minimum. This system is composed of autonomous agents which allow to model each discipline independently. They interact and cooperate with each other in order to solve discipline interdependencies. Inside the MAS, each discipline may be easily distributed and may evolve without impacting the global system.

As an MDO problem implies different disciplines, several engineers (one per discipline for instance) may have to intervene in the global optimization process of the problem. We propose that each engineer may directly interact with the system during the solving process in order to change, to test, to adapt or to add elements to the parts of the problem inherent to its discipline. This implies offering the engineers an easy way to modify their own constraints of the problem, to set specific values to some variables or change their definition domains and to automatically take these changes into account. We call this vision of MDO Integrative and Interactive Design as stated by the ID4CS project ${ }^{1}$.

Our main focus is to design the self-adaptation capabilities of the proposed system as a potentially infinite feedback loop between the system and its environment, which is typical of self-adaptive and self-organizing complex systems. As explained in [3], by using the emergence phenomena in artificial systems, our aim is to obtain a system able to cut through the search space of any problem far more efficiently than by simply dividing the problem and distributing the calculus.

In the next part (section II), we begin by reviewing existing optimization methods, both from MDO and MAS sides, and argue that they are not adapted to solve the issues we propose to tackle. Then, we present in section III a new generic agent-based modeling for continuous optimization problems, called Natural Domain Modeling for Optimization (NDMO). Using NDMO we describe in section IV an adaptive multiagent algorithm to solve continuous optimization problems, and detail in section $\mathrm{V}$ the mechanisms we introduced to handle the specificities of MDO. We present in section VI the results of our algorithm on different test cases, and finish by perspectives about future improvements based on the current work.

${ }^{1}$ Integrated Design for Complex Systems, national french project regrouping 9 academic and industrial partners, including Airbus and Snecma (Safran Group)

http://www.irit.fr/id4cs 


\section{EXISTING METHODS}

\section{A. MDO methods}

As presented in the introduction, MDO problems are highly complex continuous optimization problems. This complexity cannot be solely explained by the sheer size of the problem, since even "big" continuous optimization problems can easily be solved depending of their topology, but comes from the interactions and reciprocal dependencies of the different disciplines. As each discipline aims at modeling a part of the physical world, integrating them together will bring back some inherent non-linearities which were not present in the simplified view of each separate model. Consequently such MDO problems can truly be qualified as "complex", and finding a solution to such a problem entails necessarily to find an equilibrium among the local constraints and objectives expressed on the different parts of the system.

Classical MDO methods delegate the optimization in itself to standard optimization techniques, which must be chosen and applied by the engineer, according to his knowledge of the problem and his skills. The functioning of these methods can vary greatly. For example Multi-Disciplinary Feasible Design, considered to be one of the simplest methods [4], consists only in a central optimizer taking charge of all the variables and constraints sequentially, but gives poor results when the complexity of the problem increases [5]. Other approaches, such as Collaborative Optimization [6] or BiLevel Integrated System Synthesis [7], are said bi-level. They introduce different levels of optimization [8], usually a local level where each discipline is optimized separately and a global level where the optimizer tries to reduce discrepancies among the disciplines. However, these methods can be difficult to apply since they often require to profoundly reformulate the problem [9], and can have large computation time [5].

One of the major shortcomings of these classical methods is that they require a lot of work and expertise from the engineer to be put in practice. To actually perform the optimization process, one must have a deep understanding of the models involved as well as of the chosen method itself. This is mandatory to be able to correctly reformulate the models according to the formalism the method requires, as well as to work out what is the most efficient way to organize the models in regard to the method. Since by definition MDO involves disciplines of different natures, it is often impossible for one person to possess all the required knowledge, needing the involvement of a whole team in the process. Moreover, answering all these requirements implies a lot of work before even starting the optimization process.

\section{B. Multi-Agent Systems for Optimization}

While multi-agent systems have already been used to solve constraint satisfaction and optimization problems [10][12], the existing works mainly concern their application to Combinatorial Optimization, notably in the context of the DCOP (Distributed Constraint Optimization Problem) formalism, which usually applies to constraint optimization problems where the definition domains of the design variables are discrete and finite.

In DCOP, the agents try to minimize a global cost function (or alternatively, maximize a global satisfaction) which

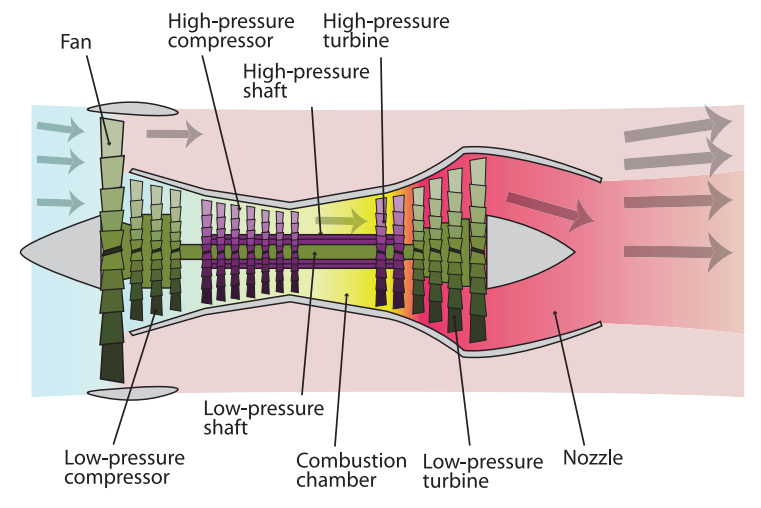

Fig. 1. Illustration of a Turbofan engine (CC SA-BY K. Aainsqatsi)

depends on the states of a set of design variables. Each design variable of the optimization problem is associated with an agent. The agent controls the value which is assigned to the variable. The global cost function is divided into a set of local cost functions, representing the cost associated with the conjoint state of two specific variables. An agent is only aware of the cost functions which involve the variable it is responsible for.

While some works successfully used DCOP in the context of continuous optimization [13], this formalism is not adequate to handle the type of problems we propose to solve here. DCOP problems are supposed to be easily decomposable into several cost functions, where the cost values associated to the variables states are supposed to be known. This major assumption does not stand for MDO problem, where the complexity of the models and their interdependencies cause this information to be unavailable in most cases. Trying to model such MDO problems with DCOP would result in a system where most agents are related to every other agent, with unknown cost functions.

Moreover, the existing agent-based optimization techniques for DCOP often present similar shortcomings to MDO methods, in the sense that they require a strong expertise to be efficiently applied [14].

\section{Problem Modeling With NDMO}

In answer to the previous shortcomings, we propose a generic approach called Natural Domain Modeling for Optimization (NDMO) that relies on a natural or intrinsic description of the problem (i.e. close to the reality being described).

To illustrate how an optimization problem is modeled, we use a simplified Turbofan optimization problem. In Fig. 1, an illustration of the principle of the turbofan can be seen. In this figure, the bypass ratio is the ratio between the air drawn in by the fan not entering engine core (which is bypassed) and the air effectively used for the combustion process. The pressure ratio is the ratio between pressure produced by the compressors and the pressure it receives from the environment.

In order to identify the elements of a generic continuous optimization model, we worked with experts from several related fields: numerical optimization, mechanics as well as 


$$
\begin{gathered}
(\operatorname{Tdm0}, s, f r)=\text { Turbofan }\left(p i \_c, b p r\right) \\
\max T d m 0 \\
\text { min } s \\
\text { subject to } \\
s \leq 155 \\
f r \geq 4
\end{gathered}
$$

(a) mathematical formulation.

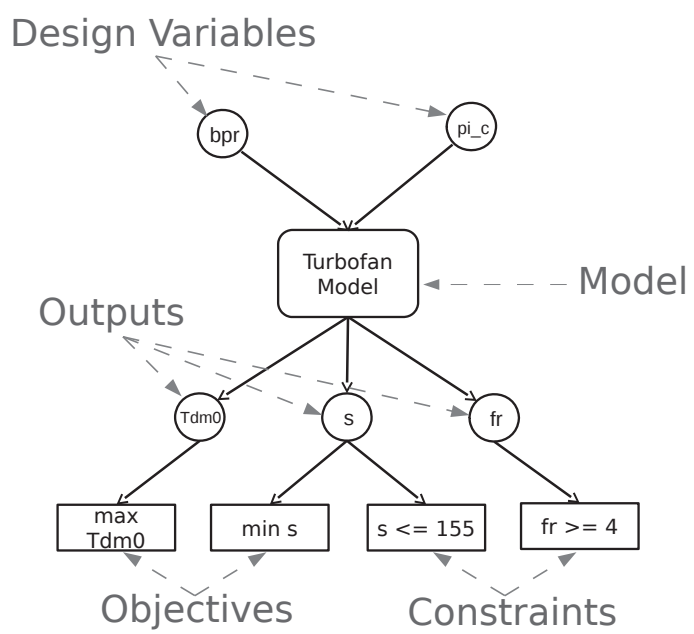

(b) corresponding entities graph.

Fig. 2. Turbofan problem.

aeronautics and engine engineers. As a result, we identified five classes of interacting entities: models, design variables, output variables, constraints and objectives.

In Fig. 2a, the analytic expression of this optimization problem is given, while in Fig. $2 b$, the problem is presented as a graph of the different entities. The design variables of this problem are pi_c and $b p r$, which indicate respectively the compressor pressure ratio and the bypass ratio of the engine. The turbofan model produces three outputs: $\operatorname{Tdm} 0, s$ and $f r$, representing respectively the thrust, fuel consumption and thrust ratio of the engine. In this problem we try to maximize the thrust and minimizing the fuel consumption while satisfying some feasibility constraints.

Let's now see in more details the roles of each of these five entities: model, variable, output, constraint and objective.

Models: In the most general case, a model can be seen as a black box which takes input values (which can be design variables or output variables) and produces output values. A model represents a technical knowledge of the relations between different parts of a problem and can be as simple as a linear function or a much more complex algorithm requiring several hours of calculation. Often some properties are known (or can be deduced) about a model and specialized optimization techniques can exploit this information. In our Turbofan example, a model entity is the Turbofan function which calculates the three outputs using the values of $b p r$ and pi_c.

Design Variables: These are the inputs of the problem and can be adjusted freely (within their defining boundaries). The goal is to find the set(s) of values for these variables that maximize the objectives while satisfying the constraints. Design variables are used by models to calculate their outputs and by constraints and objectives to calculate their current value. A design variable can be shared by several models, objectives and constraints. Keeping with our example, bpr and pi_c are the two design variables of our optimization problem.

Output Variables: These values are produced by a model, and consequently cannot be changed freely. As for the design variables, the output variables are used by models to calculate their outputs and by constraints and objectives to calculate their current value. In our example, $T d m 0, s$ and $f r$ are output variables produced by the Turbofan model.

Constraints: These are strict restrictions on some parts of the problem, represented as functional constraints defined by equalities and/or inequalities. These can be the expression of a physical constraint, or a requirement concerning the problem. Regarding the Turbofan, the two constraints are $s<=155$ and $f r>=4$.

Objectives: The goals to be optimized. In the general case, different objectives are often contradictory. The two objectives of the Turbofan problems are to maximize $T d m 0$ and to minimize $s$.

An interesting and important point is that both models, constraints and objectives involve computation. Often the most heavyweight calculus is encapsulated inside a model and the calculi concerning criteria tend to be simple equations, but this is neither an absolute requirement nor a discriminating characteristic.

The NDMO modeling aims to provide the most complete and natural representation of the problem. This modeling preserves the relations between the domain entities and is completely independent of the solving process. Since we now have a way to model optimization problems as graphs of entities, we now present the multi-agent algorithm proposed to solve them.

\section{A Multi-AGEnt System for MDO}

Based on the NDMO modeling in section III, we propose a multi-agent system where each domain entity is associated with an agent. Thus, the multi-agent system is the representation of the problem to be solved with the links and communication between agents reflecting its natural structure. It is worth emphasizing the fact that this transformation (i.e. the agentification) can be completely automatic as it is fully derived from the expression of the problem.

The local behavior of the agents has to be tailored in a way leading to an equilibrium between all the constraints and objectives expressed on the different parts of the problem.

To describe the solving process - constituted by the collective behavior of the agents - we must describe the behavior of each type of agents.

But before that, let us explain the resulting emergent behavior of the system. It basically relies on two continuous simultaneous flow of information: downward (from design variables to criteria) with new values computed by models, and upward (from criteria to design variables) with changevalue requests that drive the movements of the design variable 
in the search space. Intuitively, by emitting requests, criteria agents are "pulling" the different design variables, through the intermediary agents, in multiple direction in order to be satisfied. The system thus converges to an equilibrium between all these "forces", especially in the case of multiple contradicting criteria, which corresponds to the optimum to be found.

Methodologically, by studying how the system handles specific problems with specific characteristics, we defined different cooperation mechanisms that enable the system to work for all problems with these characteristics. Some of these mechanisms are presented in section V.

We now detail the general behaviors of our five agent types: model, variable, output, constraint and objective agents. A summary of the basic principles of each agent type is given in Algorithm 1.

Model Agent: A model agent takes charge of a model of the problem. It interacts with the agents handling its inputs (which can be variable or output agents) and the output agents handling its outputs. Its individual goal is to maintain the consistency between its inputs and its outputs. To this end, when it receives a message from one of its inputs informing it of a value change, a model agent recalculates the outputs values of its model and informs its output agents of their new value. On the other part, when a model agent receives a message from one of its output agents it translates and transmits the request to its inputs.

This translation done by the model agent consists in finding the input values corresponding to a specific desired output value, the model agent can use external optimization techniques provided by the engineer based on expert domaindependent knowledge regarding the structure of the model itself. Alternatively, a general basic mechanism based on linear interpolations is provided. It is important to emphasize that the optimizer is used only to solve the local problem of the model agent, and is not used to solve the problem globally.

Variable Agent: This agent represents a design variable of the problem. Its individual goal is to find a value which is the best equilibrium among all the requests it can receive (from models and criteria for which it is an input). The agents using the variable as input can send to it request asking to change its value. When changing value, the agent informs all agents linked to it of its new value.

To find its new value, the variable agent uses an exploration strategy based on Adaptive Value Trackers (AVT) [15]. The AVT can be seen as an adaptation of dichotomous search for dynamic values. The main idea is to change value according to the direction which is requested and the direction of the past requests. While the value varies in the same direction, the variation delta is increased so the value varies more and more. As soon as the requested variation changes, it means that the variable went past the good value, so the variation delta is reduced.

This capability to take into account a changing solution allows the variable agent to continuously search for an unknown dynamic target value. This capability is also a requirement for the system to be able to adapt to changes made by the engineer during the solving process.

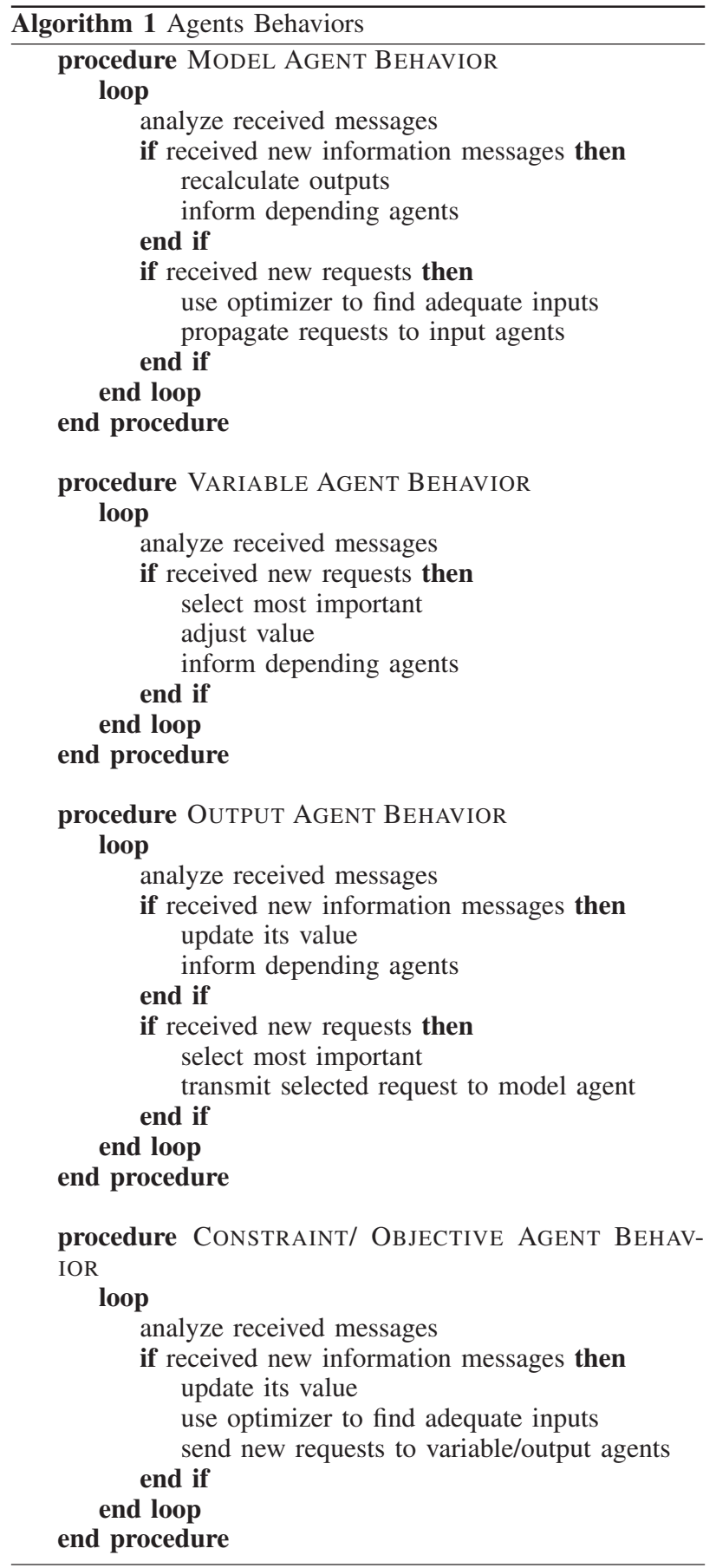


Output Agent: The output agent takes charge of an output of a model. Output agent and variable agents have similar roles, except output agents cannot directly change their value. Instead they send a request to the model agent they depend on. In this regard, the output agent act as a filter for the model agent it depends on, selecting among the different requests the ones it then transmits.

As we will see in the next section, the output agent is distinct from the variable agent in the way that it can be involved in cycles. A cycle is a situation of interdependent models (that is, models which depend on each other to calculate their outputs).

Constraint Agent: The constraint agent has the responsibility for handling a constraint of the problem. When receiving a message from one of its inputs, the agent recalculates its constraint and checks its satisfaction. If the constraint is not satisfied, the agent sends change value requests to its inputs. It should be noted that, to estimate the input values required to satisfy the constraint on its computed value, this agent employs the same technique as the model agent (i.e. an external optimizer).

Objective Agent: The objective agent is in charge of an objective of the problem. This agent sends requests to its inputs aiming to improve its objective, and recalculates the objective when receiving value changed messages from its inputs.

This agent uses an external optimizer to estimate input values which would improve the objective, as the model and constraint agents.

The most important point is that each agent only has a local strategy. No agent is in charge of the optimization of the system as a whole, or even of a subset of the other agents. Contrary to the classical MDO methods presented earlier, the solving of the problem is not directed by a predefined methodology, but by the structure of the problem itself. The emerging global strategy is unique and adapted to the problem.

\section{CoOperation Mechanisms}

In order to reach a correct equilibrium state despite the potentially complex non-linearities of the problem, we endowed the agents with three main mechanisms. They handle specific challenges related to MDO: Criticality, Simultaneous Cooperative Multi-Request Satisfaction and Cycle Handling. Two other lesser mechanisms that support the behavior of the agents are not described here (managing hidden dependencies and waiting for delayed information).

\section{A. Criticality: A Heuristic for Local Cooperation}

The design of the agents' behavior is based on cooperation. The main idea of cooperation is for agents to try to help other agents which are less satisfied than themselves, that is, which are in a more critical state than themselves. The purpose of this mechanism is to provide the agents with synthetic information regarding the parts of the system it is not able to directly perceive because of its local perception, by propagating the information in the system from agent to agent.

To evaluate this critical state of an agent as a single, comparable numerical value, a measure called criticality is used [16]. This indicator can then be transmitted to the other agents or the engineer. Facing contradictory requests, an agent can choose which request to satisfy by observing and comparing the criticalities of the senders. For example, the variable agent uses the criticality to discriminate between contradictory change requests, choosing the request from the agent which is the most critical (that is, the agent whose criticality is the highest). The strengths of this approach are its flexibility and ease of interpretation by a human. This notion of criticality as a heuristic for local cooperation was defined in the Adaptive Multi-Agent System theory [17].

In the proposed system, criticality is computed by criteria agents and is propagated in the system through their requests. We illustrate this with a constraint of the type $g(X) \leq t$, with $X$ input of the constraint, $g(X)$ the constraint equation and $t$ the threshold under which the constraint is satisfied. The basic requirements regarding the criticality of this agent is to be low when the constraint is satisfied and high when the constraint is violated. Thus, the criticality of this agent is a function of its current value and of the threshold.

To compute it, we use the function defined in Fig. 3. It takes as input $x$, the current value of the constraint. It is parameterized by $t$, the threshold, and by $\eta$ and $\epsilon$ that both regulate the shape of the function as seen in Fig. 4. Its value always varies between 0 and 1 . The $\epsilon$ can be adjusted by a domain expert, if needed: the higher it is, the faster the constraint increases in criticality. In our experiments, we used $\epsilon=0.1$ and $\eta$ was set to roughly a third of $\epsilon$, i.e. 0.03 . This function allows a smooth transition between two states and provides several interesting properties: it is continuous, differentiable, requires few parameters, is computed quickly and is relatively easy to grasp.

The criticality of the other agents is determined as follow:

- For objective agents: the criticality is set to an arbitrary constant value which must be lower than 1 . In our experiments we settled for a value of 0.5. This translates the fact that, in the general case, an objective could theoretically always be improved, but is less important to satisfy than a constraint.

- For variable, output and model agents: the criticality is set to the highest criticality among the received requests.

When the system converges to a solution, it stabilizes at a point where the maximum of the criticalities of the agents is minimized.

\section{B. Simultaneous Cooperative Multi-Request Satisfaction}

Another very common difficulty in MDO problems is the presence of multiple objectives and constraints, often contradictory. Consequently a model agent often receives contradictory requests from its output originating from different criteria. To ensure the convergence of the system towards a good solution, it is important to handle these requests in the most cooperative way.

As we presented earlier, objectives and constraints try to improve their local goals independently, without taking each other in account. During the solving, a model agent can receive contradictory requests originating from these criteria agents. In this case, the normal behavior of the model agent is to select the most critical request, disregarding the others. However in some cases, this behavior could be inefficient, as it is possible 


$$
\begin{gathered}
\text { criticality }, \eta, \epsilon(x)= \begin{cases}0 & \text { if } x<t-\epsilon, \\
\frac{-\gamma(t-x-\eta)^{2}}{2(\epsilon-\eta))}+\gamma(t-x-\eta)+\delta & \text { if } t-\epsilon \leq x \leq t-\eta, \\
\frac{\gamma(-t-x-\eta)^{2}}{2 \eta}+\gamma(-t-x-\eta)+\delta & \text { if } t-\eta \leq x \leq t, \\
1 & \text { if } x>t\end{cases} \\
\text { where } \\
\gamma=-2 / \epsilon, \\
\quad \begin{array}{l}
\gamma=\gamma(\epsilon-\eta) / 2, \\
\text { and } 0<\eta<\epsilon .
\end{array}
\end{gathered}
$$

Fig. 3. Analytical formulation of the criticality function.

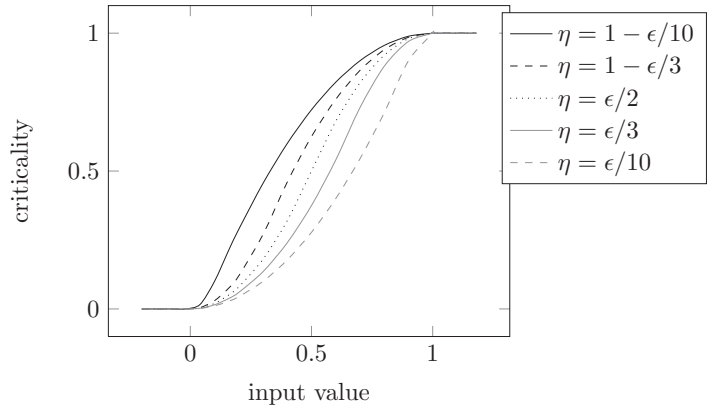

Fig. 4. Shapes of criticality function of threshold $t=1$ for $\epsilon=1$ and different

to find a set of actions (or "direction" in the search space) which would satisfy several criteria at the same time. Indeed, the outputs of a model can actually be sensitive only to a subset of its input.

The model agent is given a mechanism to estimate the correlation between each outputs and inputs, i.e. how much an output changes when a given input changes. Such a measure is only valid at a given time and is constantly revised when the model recomputes its outputs.

When sending its requests, the model agent can then for each input base its demand on the request of the most impacted output. By satisfying the requests in the most cooperative way, the model agent improves the efficiency of the system.

\section{Cycle Handling}

Another common situation in complex systems is the presence of interdependency cycles (i.e. models which depend of each other to calculate their outputs). The solution (if it exists) when such a cycle is stabilized is called the fixed point. To be able to converge towards such a point, we must introduce specific mechanisms to:

- Detect the existence of a cycle.

- Determine if the fixed point is attractive or repulsive.

- In the case of a repulsive fixed point, develop a strategy to ensure convergence towards it.

To address the first point, each message is uniquely signed to register its origin. When a variable agent sends a message, it signs the message with its unique agent "ID" and a unique sender-relative (i.e. order is only valid for a given origin) message number. The association of these two elements is the origin signature. This signature is preserved from message to message when forwarding messages and can be used to pinpoint the origin of an action in the system.

The output agents are in charge of detecting and handling cycles, as they are in the best position for being at the junction between criteria and models (or between different models).

To detect a cycle, the output agent creates a correspondence table associating to each origin the last signature it received from it. Every time a message is received, before updating this table with the new signatures, the agents checks if the signature matches with one that was already seen. If it is the case, then it means it saw twice a message pertaining to a given action in the system and that there is a cycle.

As in the general case all models are black boxes, the output agent needs to observe the evolution of its value when a cycle is detected to determine whether it is diverging or converging towards the fixed point. Because the system converges by oscillating around the solution, if the difference between successive values is decreasing, the cycle is converging towards the fixed point, else the cycle is diverging. In the case of a diverging cycle, instead of taking the new value from the model, the agent counteracts it by inverting the tendency by applying the inverse variation to its value instead of just propagating it.

\section{EXPERIMENTS}

In this section we present three test cases, Alexandrov Problem, Turbofan Problem and Viennet1, on which our system has been applied, and the experimental results we obtained. In each test case, the MAS consistently converges towards the best (or one of the best) solution.

\section{A. Alexandrov Problem}

Our first test case is inspired from an academic example taken in literature by Alexandrov et al [8]. This simple example presents some of the common characteristics of MDO problems, such as interdependent disciplines and multiple criteria. In the original article, the example was used to illustrate some properties of Collaborative Optimization, which we presented earlier, in terms of reformulation. While the paper only gave the structure of the problem, we adapted it with meaningful values and equations. The formulation of the problem is: 

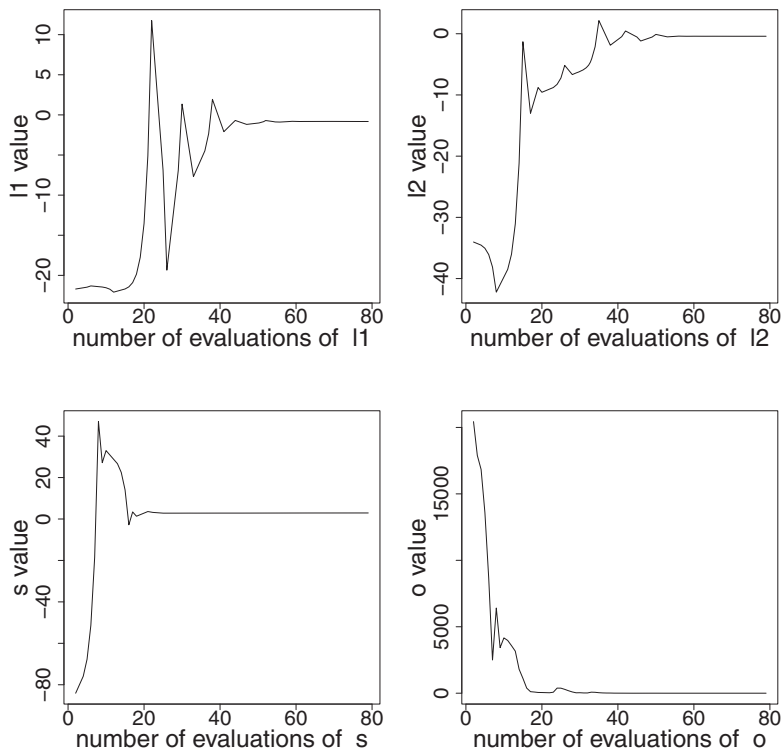

Fig. 5. Alexandrov agents behavior

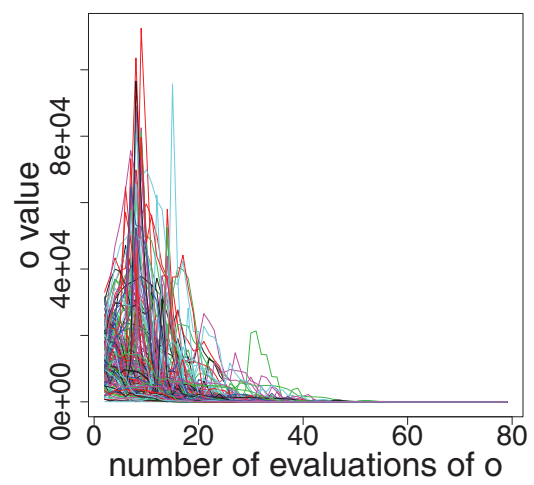

Fig. 6. Convergence of the Alexandrov objective for 100 random starting points

$$
\begin{gathered}
a_{1}=\left(l_{1}-a_{2}\right) / 2 \\
a_{2}=\left(l_{2}-a_{1}\right) / 2 \\
\text { Minimize } \frac{1}{2}\left(a_{1}^{2}+10 a_{2}^{2}+5(s-3)^{2}\right) \\
\text { subject to } \\
s+l_{1} \leq 1 \\
-s+l_{2} \leq-2
\end{gathered}
$$

In Fig. 5, the behavior of the design variables agents 11, 12 and $\mathrm{s}$, as well the evolution of the objective, can be observed on one instance of the problem with random starting points, as well as the stabilization of the system on the optimum solution.

In Fig. 6, we show the evolution of the objective over 100 iterations with starting points for each design variable randomly drawn over the interval $[-100 ; 100]$. We can see
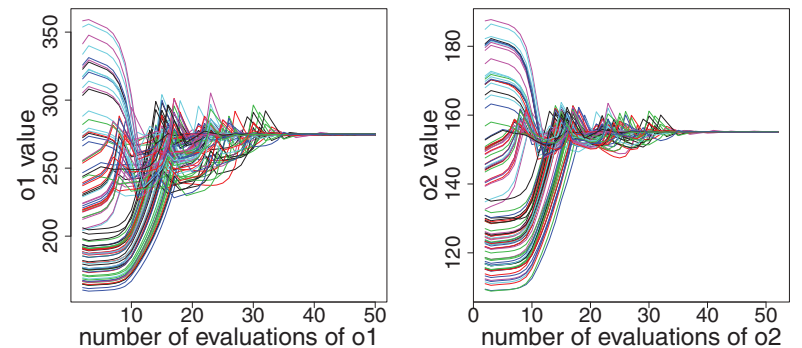

Fig. 7. Convergence of the Turbofan objectives for 100 random starting points

how the system converges towards the same optimum despite the wildly different initial conditions. We can see how the system converges towards the same optimum despite the wildly different initial conditions, showing the low sensibility of the system to initial conditions.

\section{B. Turbofan Problem}

The turbofan problem we introduced in Fig. 2 is based on a real-world optimization problem, albeit simplified for demonstration purposes, concerning the conception of a turbofan engine.

As stated before, the problem involves two design variables $p i \_c$ and bpr. pi_c is defined inside the interval [20-40] and $b p r$ inside [2-10]. The model produces three variables $T d m 0$, $s$ and $f r$. The problem has two objectives, maximizing $T d m 0$ and minimizing $s$, under the constraint $s \leq 155$ and $f r \geq 4$. The main interest and difficulty of this problem is the existence of two contradictory objectives.

In Fig. 7, we can see that the system consistently converges toward the same optimal solution, again without being disturbed by the different initial conditions.

\section{Viennet1}

The Viennet1 test case is part of a series of problems proposed in [18] to evaluate multi-criteria optimization techniques. This problem involves three objectives. Its analytical formulation is:

$$
\begin{aligned}
\text { Minimize } o 1 & =x^{2}+(y-1)^{2} \\
o 2 & =x^{2}+(y+1)^{2} \\
o 3 & =(x-1)^{2}+y^{2}+2
\end{aligned}
$$$$
\text { where } x, y \in[-4 ; 4]
$$

Fig. 8 illustrates again the convergence of the system towards a, identical correct optimum despite the different starting conditions.

\section{CONCLUSION}

We presented a generic model of numerical optimization problem and an agent-based optimization algorithm. While classical methods often have difficulties to handle complex MDO problems and require the use of specific methodologies, 

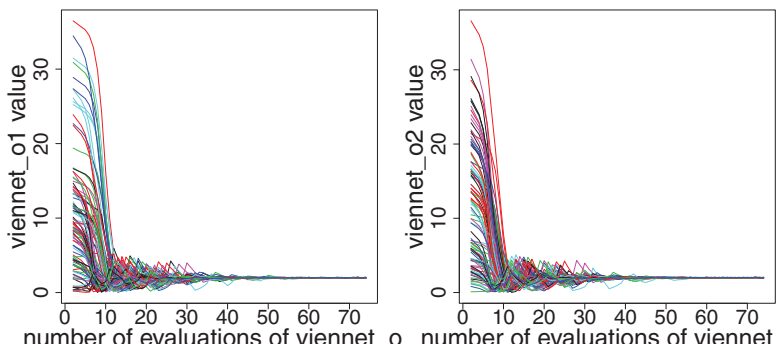

number of evaluations of viennet_on number of evaluations of viennet

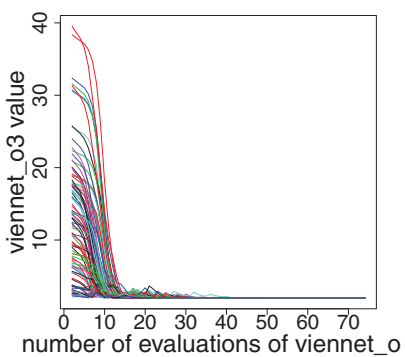

Fig. 8. Convergence of Viennet1 objectives for 100 random starting points

we distribute the problem among the agents in order to keep a low local complexity.

One of our concerns has been to facilitate the work of the engineer and allow him to express his problem in a way which is the most natural to him, instead of restricting him to a specific formulation. By analyzing the different concepts involved in the expression of an MDO problem, we extracted several atomic roles upon which we based the relations between the entities of our system. With these lowlevel entities, we are able to propose a new formalism we name NDMO. This new formalism can reconstruct a great variety of problems while mirroring their original formulation. Using this formalism, we proposed an agent-based optimization algorithm integrating MDO-specific mechanisms.

We exposed here the results of several experiments using representative problems in order to validate the soundness of our approach. We continue to work with our industrial partners in order to show the scalability of our approach on more complex real world based problems.

Our goal is to make a system that grows not only with the complexity of the problem but also with the needs of the engineer. This is why our approach can, by design, easily be interfaced with any local optimization method. In the same idea, one of our next goals is to integrate into our system the capability to handle and propagate uncertainties among the different parts of the problem.

\section{ACKNOWLEDGEMENTS.}

This work has been supported by French National Research Agency (ANR) through COSINUS program with ANR-09COSI-005 reference.

\section{REFERENCES}

[1] J. Sobieszczanski-Sobieski, R. T. Haftka, J. Sobieszczanski-sobieski, and R. T. Haftka, "Multidisciplinary aerospace design optimization: Survey of recent developments," Structural Optimization, vol. 14, pp. 1-23, 1996.

[2] G. Weiss, Ed., Multiagent Systems: A Modern Approach to Distributed Artificial Intelligence. MIT Press, 1999.

[3] G. Serugendo, M. Gleizes, and A. Karageorgos, "Self-organising systems," Self-organising Software: From Natural to Artificial Adaptation, p. 7, 2011.

[4] E. Cramer, J. Dennis Jr, P. Frank, R. Lewis, and G. Shubin, "Problem formulation for multidisciplinary optimization," SIAM Journal on Optimization, vol. 4, no. 4, pp. 754-776, 1994.

[5] S. Yi, J. Shin, and G. Park, "Comparison of mdo methods with mathematical examples," Structural and Multidisciplinary Optimization, vol. 35, no. 5, pp. 391-402, 2008.

[6] I. M. Kroo, S. Altus, R. D. Braun, P. J. Gage, and I. P. Sobieski, "Multidisciplinary optimization methods for aircraft preliminary design," AIAA 5th Symposium on Multidisciplinary Analysis and Optimization, September 1994, aIAA 1994-4325.

[7] J. Sobieszczanski-Sobieski, J. Agte, and R. Sandusky, Bi-Level Integrated System Synthesis. NASA Langley Technical Report Server, 1998.

[8] N. Alexandrov and R. Lewis, "Analytical and computational aspects of collaborative optimization for multidisciplinary design," AIAA journal, vol. 40, no. 2, pp. 301-309, 2002.

[9] R. Perez, H. Liu, and K. Behdinan, "Evaluation of multidisciplinary optimization approaches for aircraft conceptual design," in AIAA/ISSMO Multidisciplinary Analysis and Optimization Conference, Albany, NY, 2004.

[10] B. Shi and J. Liu, "Self-organizing agents for efficient sustainable resource utilization," in Proceedings of the The 2012 IEEE/WIC/ACM International Joint Conferences on Web Intelligence and Intelligent Agent Technology - Volume 02, ser. WI-IAT '12. Washington, DC, USA: IEEE Computer Society, 2012, pp. 510-517.

[11] R. Mailler, "Improving asynchronous partial overlay," in Proceedings of the The 2012 IEEE/WIC/ACM International Joint Conferences on Web Intelligence and Intelligent Agent Technology - Volume 02, ser. WI-IAT '12. Washington, DC, USA: IEEE Computer Society, 2012, pp. 9-16.

[12] A. ter Mors, C. Witteveen, C. Ipema, F. de Nijs, and T. Tsiourakis, "Empirical evaluation of multi-agent routing approaches," in Proceedings of the The 2012 IEEE/WIC/ACM International Joint Conferences on Web Intelligence and Intelligent Agent Technology - Volume 02, ser. WI-IAT '12. Washington, DC, USA: IEEE Computer Society, 2012, pp. 305-309.

[13] R. Stranders, A. Farinelli, A. Rogers, and N. Jennings, "Decentralised coordination of continuously valued control parameters using the maxsum algorithm," in Proceedings of The 8th International Conference on Autonomous Agents and Multiagent Systems-Volume 1. International Foundation for Autonomous Agents and Multiagent Systems, 2009, pp. 601-608.

[14] E. Kaddoum, "Optimization under Constraints of Distributed Complex Problems using Cooperative Self-Organization," Ph.D. dissertation, Université de Toulouse, Toulouse, France, november 2011. [Online]. Available: ftp://ftp.irit.fr/IRIT/SMAC/DOCUMENTS/ RAPPORTS/TheseElsyKaddoum_2011.pdf

[15] S. Lemouzy, V. Camps, and P. Glize, "Principles and properties of a mas learning algorithm: A comparison with standard learning algorithms applied to implicit feedback assessment," in Web Intelligence and Intelligent Agent Technology (WI-IAT), 2011 IEEE/WIC/ACM International Conference on, vol. 2, aug. 2011, pp. 228 -235.

[16] M.-P. Gleizes, "Self-Adaptive Complex Systems," in 9th European Workshop on Multiagent Systems (EUMAS 2011), Proceedings, M. Cossentino, M. Kaisers, K. Tuyls, and G. Weiss, Eds. Springer, 2012.

[17] M. Gleizes, V. Camps, J. Georgé, and D. Capera, "Engineering systems which generate emergent functionalities," Engineering EnvironmentMediated Multi-Agent Systems, pp. 58-75, 2008.

[18] R. Viennet, C. Fonteix, and I. Marc, "Multicriteria optimization using 
a genetic algorithm for determining a pareto set," International Journal of Systems Science, vol. 27, no. 2, pp. 255-260, 1996. 